\title{
Effect of clomipramine in a dog with cataplexy
}

\author{
Soo-Yeon Jeong, Min-Hee Kang, Hee-Myung Park* \\ Department of Veterinary Internal Medicine, College of Veterinary Medicine, \\ Konkuk University, Seoul 143-701, Korea \\ (Received: November 17, 2012, Revised: February 26, 2013, Accepted: March 13, 2013)
}

\begin{abstract}
A 5-year-old, castrated male, Maltese was presented with history of acute flaccid paralysis. The dog was presented with sudden loss of muscle tone and involuntary movements of hind limbs. Neurologic examination revealed reduced postural reaction in the bilateral hind limbs. MRI of brain showed moderate hydrocephalus, but other examination results were normal. Based on the characteristic episodes and examination results, canine cataplexy was suspected. Treatment was initiated with clomipramine as cataplexy control. Clinical signs resolved with 3-month medication. This case demonstrates therapeutic diagnosis of cataplexy. To the author's knowledge, this is the first report of cataplexy treating with clomipramine.
\end{abstract}

Keywords : cataplexy, clomipramine, narcolepsy

Cataplexy in dogs is relatively rare disease and manifests itself as sudden weakness of muscle tone, often triggered by positive emotional stimuli [5]. In genetically narcoleptic Doberman puppies, the mean age of onset of cataplexy is about 9 weeks, and female has more severe cataplexy than males [9].

Most of narcolepsy, which is sleep disorder, dogs experience clinical signs of cataplexy [2]. Two different pathophysiology mechanisms are involved in cataplexy and narcolepsy. Mutation in the hypocretin-receptor-2 gene causes the familial type $[7,8,10]$. This type is inherited autosomal recessive transmission and onset is young age ( $\leq 6$ months) [10]. The other is sporadic type caused by loss of the hypocretin ligand, which is an excitatory peptide neurotransmitter made in the hypothalamus [4]. Onset of age is wide range as compared with familial type [10].

In human medicine, generalized cataplexy causes complete collapse of patients, whereas partial cataplexy affects only the face, voice or arms. In severe cases, respiratory and extraocular muscles could be attacked [6]. In veterinary medicine, cataplexy attack may be affect all muscles or restricted to head, trunk or certain limbs [4]. According to the previous reports [4], most cataplexy attacks initiated in the hind legs and affected bilateral.

This report describes therapeutic diagnosis and successful use of clomipramine in the treatment of dog with cataplexy.

A 5-year-old, castrated male, Maltese dog was presented to history of flaccid paralysis 1 to 2 times a week. The dog fell to the ground completely atonic, and bilateral hind limbs moved involuntary. There was no loss of consciousness. This neurological signs observed 2 months prior to the presentation, and episode lasted a few seconds to $2 \mathrm{~min}$. A severe episode also occurred with loss of consciousness, elicited by excitement. Previous administration of nonsteroidal antinflammatory drug (Firocoxib, Previcox; Merial, France) by the referring veterinarian for pain control didn't improve the clinical signs. Physical examination revealed intermittent hind limbs weakness when physical manipulation of both patellar (grade I/IV medial patellar luxation), but no other abnormalities were found. The hemogram showed stress leukogram $(21,030 / \mu \mathrm{L}$, reference range, $6,000 \sim 17,000 / \mu \mathrm{L})$, and serum chemistry profiles were within normal limits. There were no remarkable abnormalities in the thoracic, abdominal and accurately positioned hind limb radiographs. Electrocardiography was also examined for exclusion of possibility of cardiogenic episode, and result had no remarkable findings. To rule out myasthenia gravis and neurologic sign induced by canine distemper virus infection, laboratory examinations were performed, and acetylcholine receptor antibody concentration was within reference range $(0.02 \mathrm{nmol} / \mathrm{L}$, reference range $0.0 \sim 0.6 \mathrm{nmol} / \mathrm{L}$ ), and canine distemper polymerase chain reaction (PCR) of cerebrospinal fluid (CSF) sample result was negative. Result of food-elicited cataplexy test (FECT) for identifying cataplexy was not revealed unusual outcome. On neurologic examination, the dog presented reduced postural reaction in the bilateral hind limbs, and this result could be suspected lesion of 4th lumbar vertebra to 3rd sacral vertebra. However, lumbosacral region was normal at magnetic

*Corresponding author

Tel: +82-2-450-4140, Fax: +82-2-444-4396

E-mail: parkhee@konkuk.ac.kr 


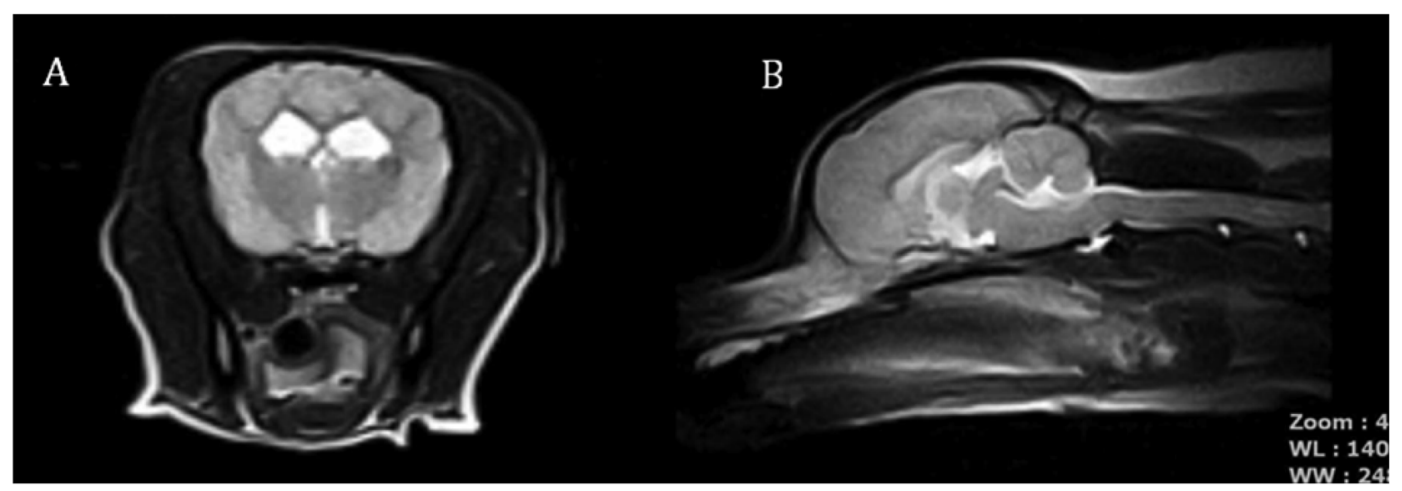

Fig. 1. Magnetic resonance imaging (MRI) of brain (A\&B). Transverse view of $T 2$ weighted image showed no remarkable findings except for moderate hydrocephalus (Ventricle/Brain ratio : 21\%) (A) Moderate extension of ventricle of the brain is also presented in sagittal view of T2 weighted image (B).

resonance imaging (MRI). Additionally, MRI for brain lesion was conducted for finding intracranial lesion, and showed moderate hydrocephalus (Ventricle/Brain ratio: 21\%, reference range: $0 \sim 14 \%$ ) (Fig. 1). Electroencephalography (EEG) and hypocretin concentration of CSF examination were recommended, but the owner declined further investigation.

Initially, the dog was prescribed furosemide $(1 \mathrm{mg} / \mathrm{kg}$ PO, q 12; Handok, Korea) and omeprazole $(0.7 \mathrm{mg} / \mathrm{kg}$ PO, q 24; Sin-Il, Korea) for the treatment of hydrocephalus. However, the treatment was discontinued a week later because the frequency of the clinical sign was increased.

Changing to other treatment, clomipramine $(2 \mathrm{mg} / \mathrm{kg}$ PO, q 12; Myung-In, Korea), was started as cataplexy control in this dog. After clomipramine with 3 weeks application, clinical signs resolved. During management, client failed to force the dog to take medicine 2 times. At that time, clinical signs were rebounded. And, after re-prescription of clomipramine, clinical sign still has not showed for 3 months (Fig. 2).

Definite diagnosis of cataplexy is not established in dogs. In cataplexy dogs, physical examination, neurologic examination, basic clinical pathologic and imaging studies are typically within normal range [4]. Generally, the diagnosis is based on dog's clinical sign and concentration of hypocretin in CSF, which is decreased in cataplexy dogs $(<100 \mathrm{pg} / \mathrm{mL}$; reference range, $200 \sim 350 \mathrm{pg} / \mathrm{mL}$ ) [10]. The FECT can be used to assess the evaluation of clinical sign. This test can be obtained number of attacks (NA) and elapsed time in collapse (ET), as well as duration of each cataplectic attack [5, 10].

Cataplexy and narcolepsy must be distinguished from syncope, epilepsy, metabolic disorders and myasthenia gravis [4]. And, withdrawal of medication abruptly may lead to cataplexy rebound, which can be helpful for diagnosis.

In this case, to establish a diagnosis, radiography, several laboratory examinations and MRI were performed. Paininduced sign could be ruled out by physical examination, history of medication. Also, acetylcholine receptor antibody concentration result and canine distemper PCR result provided that the dog didn't have myasthenia gravis, and infect canine distemper virus. FECT was attempted with atropine

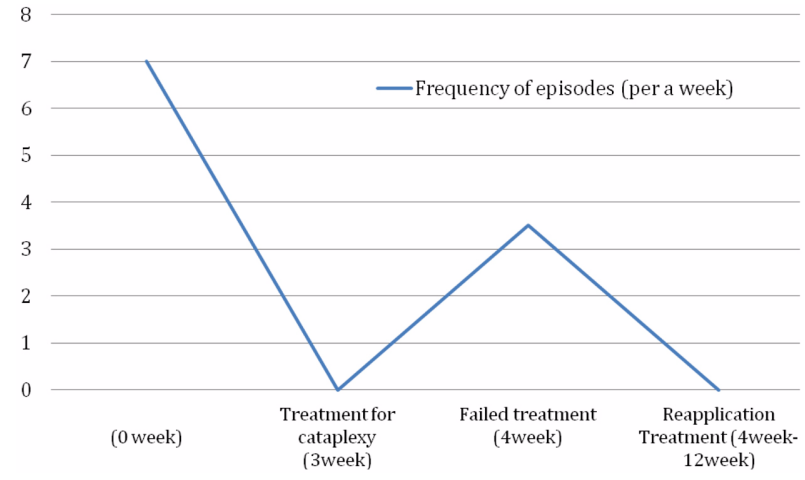

Fig. 2. Frequency of episodes for 3 months treatment. Frequency of clinical signs decreased with proper administration of clomipramine whereas failing of administration resulted in relapse of clinical sign. After reapplication treatment, clinical signs still have not shown.

$0.04 \mathrm{mg} / \mathrm{kg}$ IV treatment, but clinical sign was not presented, because the dog was afraid of hospital environment, not triggered excitement. MRI result showed the patient had moderate hydrocephalus. However, the fact that symptoms persisted after prescription of hydrocephalus showed that clinical signs was not related hydrocephalus. For therapeutic diagnosis, the dog was administrated clomipramine. The response of drug was favorable, partial seizure like episode could be excluded. Based on the examinations and therapeutic evaluation, this patient was diagnosed as cataplexy. Loss of consciousness episode was also considered as narcolepsy. Having a relapse of symptom, when the dog stopped taking his medication, supported the cataplexy diagnosis.

In veterinary medicine, various managements are followed in cataplexy treatment. Fluoxetine $(1.5 \mathrm{mg} / \mathrm{kg}$ and $3.0 \mathrm{mg} / \mathrm{kg})$ and imipramine $(1.0 \mathrm{mg} / \mathrm{kg})$ took effect on narcoleptic dog with cataplexy [1]. Immunosuppressive and anti-inflammatory agents, which consist of methylprednisolone $(1.0 \mathrm{mg} / \mathrm{kg}$, PO, $1 \times /$ day $)$, methotrexate $(0.3 \mathrm{mg} / \mathrm{kg}, \mathrm{PO}, 1 \times /$ week $)$ and azathioprine $(1.0 \mathrm{mg} / \mathrm{kg}, \mathrm{PO}, 2 \times /$ day $)$, were also tried, and onset time of symptom was doubled [2]. Intravenous and 
intracerebroventricular hypocretin therapy were administrated in sporadic type patients, but did not improve symptoms [5].

Clomipramine is known to be effective in managing cataplexy in human medicine [1]. However, effect of clomipramine treatment in cataplexy dogs has not been proven and the drug dosage was not determined. One report described that clomipramine was not effective in cataplexy dogs [1]. But, according to the human literature, the cataplexy patients showed striking amelioration of clinical signs [11].

Tricyclic antidepressants (TCAs) increase the activity of amines such as serotonin (5-HT) by blocking their reuptake into presynaptic neurons. Especially clomipramine was reported that is the most effective drug among the TCAs, in human medicine. According to this study, clomipramine is relatively selective for blocking serotonin reuptake, compared to the other TCAs, such as imipramine, desipramine and protriptyline [6]. For these reasons, the dog was tried to be prescribed clomipramine $2 \mathrm{mg} / \mathrm{kg}$ PO q $12 \mathrm{~h}$. The chosen dose based on the commonly used dose of clomipramine for the other purpose in veterinary medicine [3]. In a human medicine study, there was reported that after $6 \sim 8$ months of clomipramine medication, tolerance can develop, so the dose needs to be increased [6]. There were no specific side effects of the clomipramine and management of the cataplexy attack with clomipramine was successful for 3 months in this dog. In present, the dog is being monitored in developing a tolerance.

In conclusion, this report describes a diagnosis and management of cataplexy in a dog. Through rule out diagnostic approach and therapeutic evaluation, this dog was diagnosed as cataplexy. In addition, this is first case report described oral clomipramine in controlling cataplexy dog.

\section{Acknowledgments}

This work was supported by the National Research Foundation of Korea (NRF) grant funded by the Korea government (MEST) (No.20100018275).

\section{References}

1. Babcock DA, Narver EL, Dement WC, Mitler MM. Effects of imipramine, chlorimipramine, and fluoxetine on cataplexy in dogs. Pharmacol Biochem Behav 1976, 5, 599-602.

2. Boehmer LN, Wu MF, John J, Siegel JM. Treatment with immunosuppressive and anti-inflammatory agents delays onset of canine genetic narcolepsy and reduces symptom severity. Exp Neurol 2004, 188, 292-299.

3. Donald CP. Plumb's veterinary drug hand book, 6th ed. pp. 287-288, Blackwell, Iowa, 2008.

4. Etienne C. Clinical veterinary advisor: Dogs and Cats. p.744, Mosby, Missouri, 2011.

5. Fujiki N, Yoshida Y, Ripley B, Mignot E, Nishino S. Effects of IV and ICV Hypocretin-1 (orexin A) in hypocretin recpetor-2 gene mutated narcoleptic dogs and IV hypocretin-1 replacement therapy in a hypocretin-ligand-deficient narcoleptic dog. Sleep 2003, 26, 953-959.

6. Houghton WC, Scammell TE, Thorpy M. Pharmacotherapy for cataplexy. Sleep Med Rev 2004, 8, 355-366.

7. Hungs M, Fan J, Lin L, Lin X, Maki RA, Mignot E. Identification and functional analysis of mutations in the hypocretin (orexin) genes of narcoleptic canines. Genome Res 2001, 11, 531-539.

8. Lin L, Faraco J, Li R, Kadotani H, Roger W, Lin X, Qiu X, de Jong PJ, Nishino S, Mignot E. The sleep disorder canine narcolepsy is caused by a mutation in the hypocretin (orexin) receptor 2 gene. Cell 1999, 98, 365-376.

9. Riehl J, Nishino S, Cederberg R, Dement WC, Mignot E. Development of cataplexy in genetically narcoleptic Dobremans. Exp Neurol 1998, 152, 292-302.

10. Schatzberg SJ, Cutter-Schatzberg K, Nydam D, Barrett J, Penn R, Flanders J, deLahunta A, Lin L, Mignot E. The effect of hypocretin replacement therapy in a 3-yearold Weimaraner with narcolepsy. J Vet Intern Med 2004, 18, 586-588.

11. Shapiro WR. Treatment of cataplexy with clomipramine. Arch Neurol 1975, 32, 653-656. 